\title{
Model of single-electron decay from a strongly isolated quantum dot
}

\author{
J. Martorell \\ Departament d'Estructura i Constituents de la Materia, Facultat Física, University of Barcelona, Barcelona 08028, Spain \\ D. W. L. Sprung and P. A. Machado \\ Department of Physics and Astronomy, McMaster University, Hamilton, Ontario L8S 4M1, Canada \\ C. G. Smith \\ Semiconductor Physics Group, Cavendish Laboratory, Madingley Road, Cambridge CB3 OHE, United Kingdom
}

(Received 1 May 2000; published 9 January 2001)

\begin{abstract}
Recent measurements of electron escape from a nonequilibrium charged quantum dot are interpreted within a two-dimensional (2D) separable model. The confining potential is derived from 3D self-consistent PoissonThomas-Fermi calculations. It is found that the sequence of decay lifetimes provides a sensitive test of the confining potential and its dependence on electron occupation.
\end{abstract}

DOI: 10.1103/PhysRevB.63.045325

PACS number(s): 73.21.-b, 85.35.Be, 71.10.-w

\section{INTRODUCTION}

In a recent experiment, ${ }^{1}$ a strongly isolated quantum dot was charged with excess electrons, and their sequential escapes were recorded over a one-hour time period. This was repeated 150 times to obtain a statistical distribution of decay times. The dot is formed in an electron gas located at a depth of $70 \mathrm{~nm}$ in a GaAs- $\mathrm{Al}_{x} \mathrm{Ga}_{1-x} \mathrm{As}$ heterostructure. Its shape is defined by electrostatic confinement using a set of gates, as sketched in the inset to Fig. 1. The gate voltages were ramped up quickly, so that the dot retained a sizable number of excess electrons when it was well isolated from the surrounding electron gas. The observations correspond to sequential tunneling of (seven) electrons from the dot to the surroundings. The lifetimes extracted from the escape times distribution $^{1}$ are shown in Fig. 1. A striking quasilinear dependence of the logarithm of the lifetime on electron number is apparent.

Sequential decays have been known and studied for over a century in the context of nuclear physics. The combined instances of $\alpha$ and $\beta$ decays from the heaviest elements are responsible for most natural radioactivity. The description of $\alpha$ decay in terms of tunneling of $\alpha$ particles through a confining potential dates back to the 1920s (Gamow, ${ }^{2}$ and Condon and Gurney ${ }^{3}$ ). Although the basic nature of the decay as a barrier penetration is well understood, accurate predictions for radioactive lifetimes are difficult because the process by which the escaping $\alpha$ particle is preformed within the nucleus requires an understanding of four-body correlations. As a result, it is impossible to deduce accurate information on the barrier shape. Nevertheless, $\alpha$-particle decays have provided useful information on nuclear radii and the range and gross features of the nuclear interaction. ${ }^{4}$

It has become commonplace to say that a quantum dot is an artificial atom, but in fact the self-consistent potential confining electrons in a large dot has more in common with the mean field potential in a heavy nucleus: flat in the interior, with abrupt walls. An artificial nucleus is a more apt description, as will become clear in this paper. Indeed, the detection of sequential decays from an isolated quantum dot is a more favorable situation for study of the decay process, as the question of preforming the electron does not arise. Hence, we can more confidently test our knowledge of the confining barriers for electrons, as well as the profile, and dependence on occupation number, of the dot potential. We will analyze these aspects in this work, and show that these measurements of the lifetimes of "radioactive quantum dots" introduce new constraints on our ability to model their structure.

The present experiment has another significant advantage over nuclear decays: instead of counting incoherent decays from a large sample of identical nuclei, here a single dot is involved, and the correlation between consecutive events can be analyzed. In addition, it should be possible to design the shape, density, and excitation energy of the dot within rather broad margins, so that future experiments on mesoscopic systems will be much more flexible than those in nuclear systems, where only those nuclei existing in nature, or created in sufficient numbers, can be studied. Thus, the study of electron decays from a quantum dot has the potential to reveal new features of the tunneling process. This is a topic of

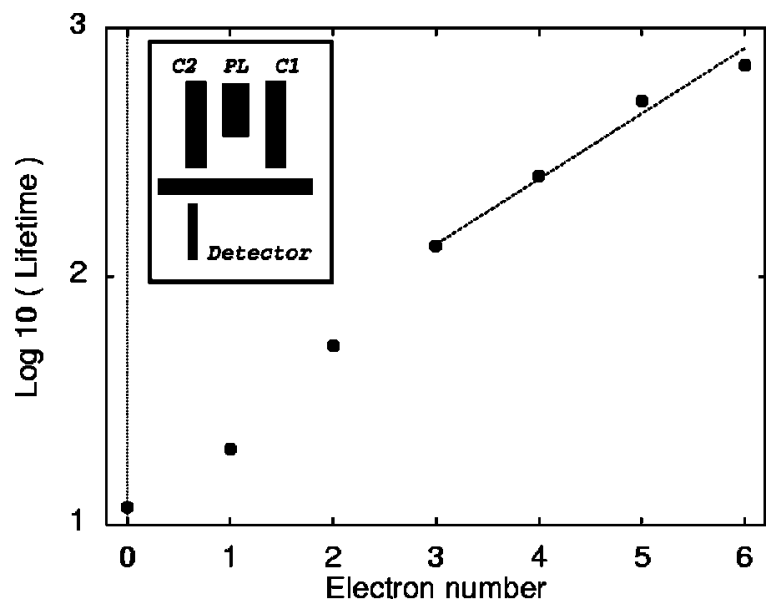

FIG. 1. Experimental lifetimes (in seconds) extracted from the decay sequences, as reported Ref. 1 . The inset shows the gate arrangement that defines the dot. 
currently renewed interest: see for example van Dijk and Nogami. ${ }^{5}$ The type of simple model developed in this paper can be of great utility in such future studies.

In this work we will describe the decay process using analytic models that incorporate characteristics of the confinement potential extracted from realistic numerical simulations. As the dot contains about 300 electrons, PoissonThomas-Fermi calculations should be adequate to describe the electron density and the confining potential of the dot. With these in hand we have developed accurate analytic approximations for the confining potential that allow us to construct an envelope approximation wave function for the electrons in the dot, and to compute the electron lifetimes from a fully quantal expression for the transmission amplitude across the barrier.

Previous works that model a quantum dot have been concerned with the wave functions of confined states in the dot, the electron density distribution, and the shape of the confining potential. For such purposes, only the inside of the barrier matters. It is when one looks at the escape of electrons from the dot that the barrier height, its width, and shape become important; these are the features explored in this paper. In Sec. II we describe the development of our model, while in Sec. III we discuss the results for the sequence of lifetimes and compare them with experiment. Some details are relegated to two Appendixes.

\section{MODELING OF ISOLATED DOT DECAYS}

\section{A. Framework}

The Poisson-Thomas-Fermi modeling is described in more detail elsewhere ${ }^{6-8}$ so here we list only the main steps:

(1) First, Poisson-Schrödinger (PS) and Poisson-ThomasFermi (PTF) simulations as described in Ref. 7 are performed for the ungated heterostructure. Our inputs for the PS simulation are the thickness and composition of each layer in the heterostructure, and the dopant concentration in the donor layer. From these we predict the density of the 2D electron gas (2DEG). The only adjustable parameter is the donor ionization energy, which is set to be $e \Phi_{i}=0.12 \mathrm{eV}$, in order to reproduce the measured 2DEG density, $n_{e}$ $=2.74 \times 10^{11} \mathrm{~cm}^{-2}$. For the simpler Poisson-Thomas-Fermi scheme we employ a common relative permitivity $\varepsilon_{r}=12.2$ for all layers of the heterostructure, which, combined with the parameters already used for the PS simulation, also reproduces the experimental $n_{e}$. After this " fitting" the model has no other free parameters.

(2) For the gated structure we use the gate layout and voltages of the experiment. To solve the Poisson equation for the gated heterostructure one has to impose as a boundary condition the value of the electrostatic potential on the exposed surface of the heterostructure and on the gates. We assume Fermi level pinning and choose the energy of the surface states as the zero of the energy scale. In this convention, the conduction band edge is set at $e V_{s}=0.67 \mathrm{eV}$ on the exposed surface. Under each gate the conduction band is set at $e V_{m s}+e V_{g}$, where $V_{g}$ is the gate voltage and the metal semiconductor contact potential, $e V_{m s}$, is taken as $0.81 \mathrm{eV} .{ }^{9}$ The electrostatic potential due to the gates is then

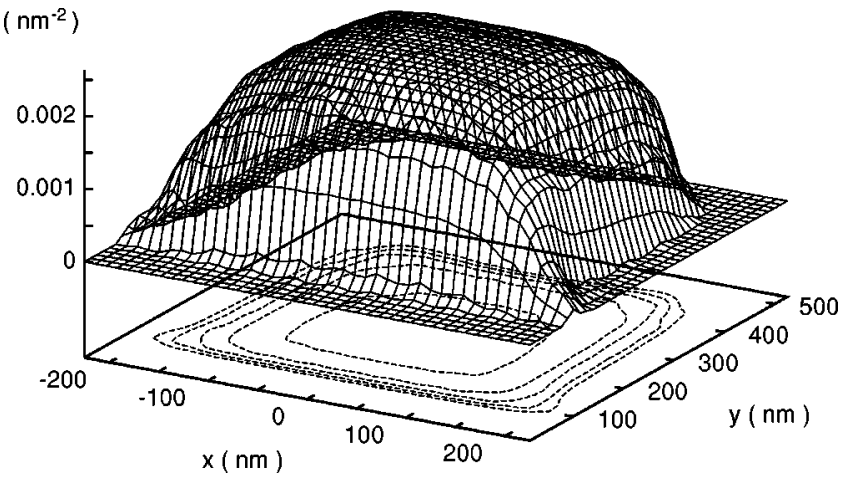

FIG. 2. The two-dimensional PTF density, $n_{e}(x, y)$, for a dot in equilibrium with the surrounding 2DEG.

computed using semianalytic expressions based on the work of Davies and co-workers. ${ }^{10,11}$ Added to this are (a) the Coulomb potential (direct term) between the electrons, and a mirror term which imposes the boundary conditions at the surface, and (b) the contribution from the fully ionized donor layer and its mirror term (see Sec. II A of Ref. 8 for details of a similar example). We neglect exchange and correlation effects, which are small.

(3) The connection between the confining potential defined by the conduction-band edge and the electron density is completed by using the Thomas-Fermi approximation at zero temperature:

$$
\rho_{e}(\vec{r})=\frac{1}{3 \pi^{2}}\left(\frac{2 m^{*}}{\hbar^{2}}\left[E_{F}-e V(\vec{r})\right]\right)^{3 / 2} .
$$

The PTF iteration is performed starting from the ungated heterostructure densities as trial values.

\section{B. Equilibrium dot}

As a first step, we examine the dot in its final state after all the excess electrons have escaped. This corresponds to a PTF simulation with the same Fermi level, $E_{F, d o t}=0$, for the electrons in the dot and in the 2DEG outside the barriers. The gate voltages are taken from Ref. 1 as $V_{P L}$ $=-0.40 \mathrm{~V}, V_{C 1}=V_{C 2}=-0.44 \mathrm{~V}$, and $V_{H}=-0.7 \mathrm{~V}$. The predicted PTF 3D electron distribution $\rho_{e}(x, y, z)$ is more conveniently visualized in terms of a projected $2 \mathrm{D}$ density:

$$
n_{e}(x, y)=\int_{z_{j}}^{\infty} \rho_{e}(x, y, z) d z,
$$

where $z_{j}$ is the junction plane. The $n_{e}(x, y)$ distribution, shown in Fig. 2, has an approximately rectangular boundary, and its maximum value is close to the 2DEG density of the ungated heterostructure. In this calculation the dot contains 286 electrons.

\section{Dot with excess electrons}

To study these configurations we set the Fermi level inside the dot, $E_{F, \text { dot }}$, higher than its value outside the barriers, $E_{F, 2 D E G}=0$. We can do so because the dot is well pinched off from the surrounding electron gas. We ran PTF 


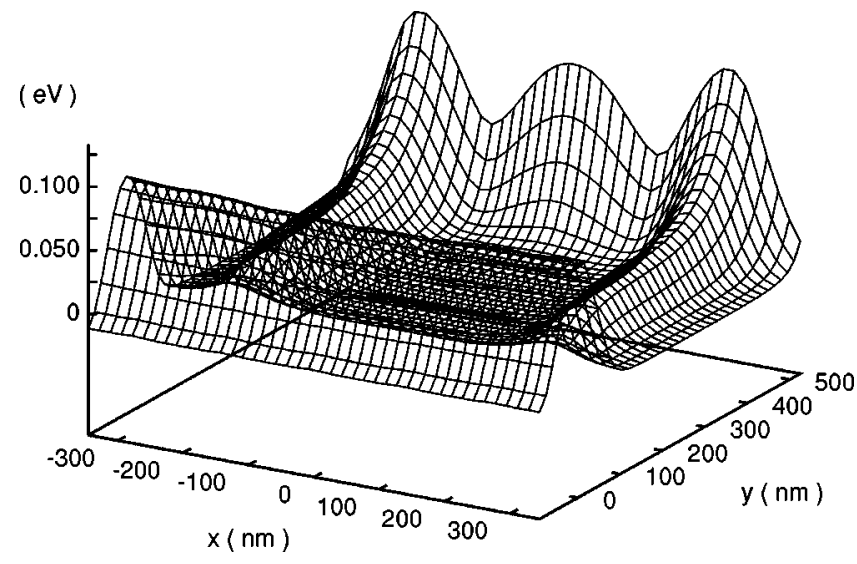

FIG. 3. Two-dimensional confining potential, $U_{P T F}(x, y)$ for the dot in Fig. 1.

simulations with equally spaced values for $E_{F, \text { dot }}$ running from 0 to $17.5 \mathrm{meV}$ in steps of $2.5 \mathrm{meV}$. The occupation $Q$ of the dot increases linearly with $E_{F, \text { dot }}$ at the rate 2.75 electrons per meV, giving occupations $286 \leqslant Q \leqslant 334$.

The simulations also produce the confining potential for the electrons in the dot, $e V(x, y, z)$. To reduce this to a twodimensional function, $U(x, y)$, we take a weighted average over the density profile in the $z$ direction:

$$
U_{P T F}(x, y)=\frac{\int_{z_{j}}^{\infty} e V(x, y, z) P(z) d z}{\int_{z_{j}}^{\infty} P(z) d z},
$$

where

$$
P(z)=\int_{\Omega} \rho_{e}(x, y, z) d x d y .
$$

Here the domain of integration $\Omega$ is a rectangle in the $x y$ plane, which extends a short distance into the surrounding electron gas $\left[\right.$ from $\left(x_{l}, y_{l}\right)=(-510 \mathrm{~nm},-255 \mathrm{~nm})$ to $\left.\left(x_{r}, y_{r}\right)=(510 \mathrm{~nm}, 255 \mathrm{~nm})\right]$. This includes an area outside the dot where the 2DEG is still depleted by the gates. Although the computed $V(x, y, z)$ is not separable, previous experience with Poisson-Schrödinger simulations of wires ${ }^{8,12}$ and circular dots has shown us that the factorization ansatz leads to very good approximations when the $z$ degree of freedom is integrated out as in Eq. (3). This prescription to construct the 2D potential avoids the type of ad hoc assumptions often made.

In Fig. 3 we show the $U_{P T F}(x, y)$ corresponding to the equilibrium dot of Fig. 2. As expected from the gate layout shown in the inset to Fig. 1, it has two very high barriers running parallel to the $x$ axis, one centered at $y=0$ and the other that begins with a steep rise at $y \simeq 400 \mathrm{~nm}$ (and shows clearly the mark of the three-fingered gate layout labeled $\mathrm{C} 1$, $\mathrm{C} 2$, and PL for plunger in Fig. 1). Tunneling across these barriers is negligible. In addition there is a symmetric pair of barriers running parallel to the $y$ axis, with maxima at $x$ $\simeq \pm 238 \mathrm{~nm}$ through which the electrons do tunnel. In the interior, the potential is practically constant. Although these $x$ barriers have somewhat increasing height with increasing

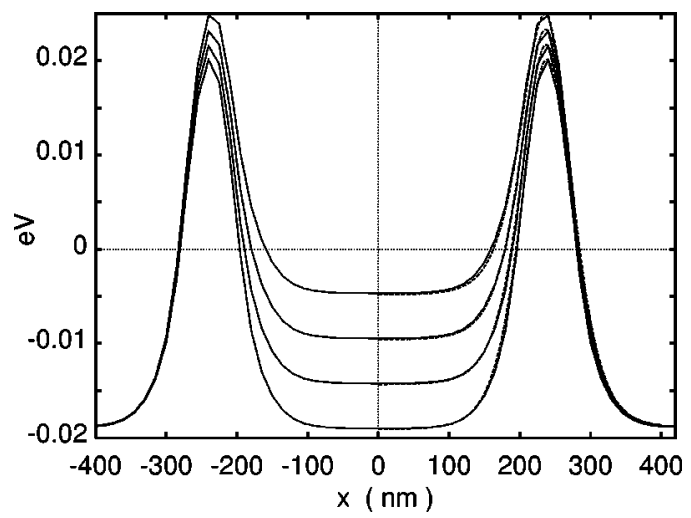

FIG. 4. Continuous lines: sections at $y=200 \mathrm{~nm}$ of the $U_{P T F}(x, y)$ corresponding to $E_{F, d o t}=0.0,0.005,0.010$ and 0.015 $\mathrm{eV}$. Dashed lines: analytic parametrization for $U(x)$ as described in the text (the latter shown only for $x>0$ for clarity).

$y$, the rectangular shape of the potential suggests using a separable approximation in Cartesian coordinates:

$$
U_{P T F}(x, y) \simeq U_{s}(x, y)=U(x)+W(y) .
$$

We will interpret the experimental decay data using this separability ansatz. For the $W(y)$ barriers, which are basically impenetrable, we use two simple models described below. As a guide to a realistic choice for the $x$-dependent term we examine in Fig. 4 the profiles of $U_{P T F}(x, y)$ at a fixed value of $y=200 \mathrm{~nm}$ in the middle of the dot. The profiles shown cover a range of occupations of up to 40 excess electrons. In this range, the potential at the dot center increases linearly with $Q$, according to

$$
U_{0}=0.347 Q-118.4 \mathrm{meV} .
$$

At large distances outside the dot, $U_{\infty}=-18.8 \mathrm{meV}$ is constant. Similarly, the location of the barrier maximum and its height can be parametrized as

$$
\begin{gathered}
x_{b}=238-\frac{Q-286}{16} \mathrm{~nm}, \\
U_{b}=0.117 Q-13.4 \mathrm{meV} .
\end{gathered}
$$

Note that $d U_{b} / d Q \approx \frac{1}{3} d U_{0} / d Q$ reflects the decrease of the screened Coulomb repulsion away from the center of the dot. Furthermore, we have found that the $x$ dependence can be very well reproduced (see Fig. 4) using the following analytic model:

$$
\begin{aligned}
U(x) & =U_{b}+U_{M F}(x), \quad x>0, \\
& =U(-x), \quad x<0,
\end{aligned}
$$

where

$$
U_{M F} \equiv U_{c} \frac{\sinh ^{2}\left(\frac{x-x_{b}}{w_{b}}\right)}{\cosh ^{2}\left(\frac{x-x_{b}}{w_{b}}-\mu\right)} .
$$


This potential form has the great advantage that the transmission coefficient for $U_{M F}$ is known analytically. ${ }^{13} U_{M F}$ is an asymmetric barrier that takes one value for $x \ll x_{b}$ and another value for $x \gg x_{b}$ :

$$
\begin{gathered}
U_{M F}\left(x_{b}\right)=0, \\
U_{M F}(\infty) \equiv \lim _{x \rightarrow \infty} U_{M F}(x)=U_{c} e^{2 \mu}, \\
U_{M F}(-\infty) \equiv \lim _{x \rightarrow-\infty} U_{M F}(x)=U_{c} e^{-2 \mu} .
\end{gathered}
$$

The parameters $U_{b}, U_{c}, \mu, x_{b}$, and $w_{b}$ allow one to fit the barrier height, the potential floors inside and outside the dot, the barrier spacing, and the barrier width. Since the barriers are spread quite far apart, in practice $x_{b} \gg w_{b}$, so $U_{M F}(x$ $=0) \approx U_{M F}(-\infty)$. In this case,

$$
\begin{gathered}
U_{0} \equiv U(0) \approx U_{b}+U_{c} e^{-2 \mu}, \\
U_{\infty} \equiv \lim _{x \rightarrow \infty} U(x)=U_{b}+U_{c} e^{2 \mu} .
\end{gathered}
$$

Then we can solve for

$$
\begin{gathered}
\mu=\frac{1}{4} \ln \left(\frac{U_{b}-U_{\infty}}{U_{b}-U_{0}}\right), \\
U_{c}=-\left(U_{b}-U_{0}\right) e^{2 \mu} .
\end{gathered}
$$

To determine the parameters appearing in Eq. (8), we take the values of the PTF potential at the origin, $U_{0}$, well beyond the barrier, $U_{\infty}$, and the value $U_{x_{b}}$ at the barrier maximum $x=x_{b}$, and then plot $U(x)$ to find the best $w_{b}$, which turned out to be $48 \mathrm{~nm}$. This gives a convenient analytic form for the confining potential, motivated by PTF simulations, whose transmission coefficient is

$$
T=\frac{2 \sinh \left(\pi k_{+}\right) \sinh \left(\pi k_{-}\right)}{\cosh \left[\pi\left(k_{+}+k_{-}\right)\right]+\cosh (\pi \beta)},
$$

where

$$
\begin{gathered}
k_{-/+}=\sqrt{\frac{2 m^{*}}{\hbar^{2}}\left(E-U_{0 / \infty}\right) w_{b}^{2}}, \\
\beta=\sqrt{\frac{2 m^{*}}{\hbar^{2}}\left(2 U_{b}-2 U_{c}-U_{0}-U_{\infty}\right) w_{b}^{2}-1 .}
\end{gathered}
$$

\section{Barrier shape $W(y)$}

In Fig. 5 we examine a section of $U_{P T F}(x=0, y)$ through the center of the dot. We use two approximate models, the simplest one being an infinite square well, of width $w_{y}$ $\approx 350 \mathrm{~nm}$. The slightly fancier model is a truncated harmonic oscillator:

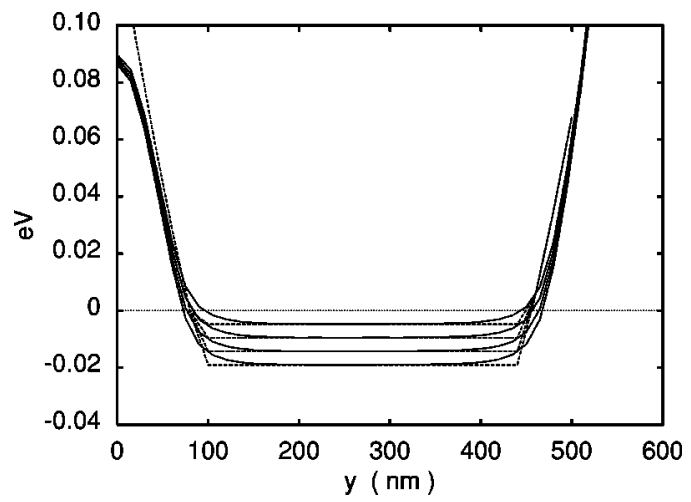

FIG. 5. Continuous lines: sections at $x=0 \mathrm{~nm}$ of the $U_{P T F}(x, y)$ for the same $E_{F, \text { dot }}$ as in Fig. 3. Dashed lines: analytic parametrization of $W_{\text {tho }}(y)+U_{0}$ as described in the text.

$$
\begin{aligned}
W_{\text {tho }}(y) & =0 \quad \text { (flat bottom) } \\
& =-0.13+\frac{1}{2} k_{y}\left(y-y_{0}\right)^{2} \quad \text { (walls). }
\end{aligned}
$$

with $y_{0}=238 \mathrm{~nm}$ and $k_{y}=7.35 \times 10^{-6} \mathrm{~nm}^{-2}$. As can be seen in Fig. 5 this parametrization (plus the constant term $U_{0}$ ) reproduces the main features of the $x=0$ sections of the PTF potentials.

By combining Eqs. (6) to (14) we determine a separable analytic potential model for the dot containing a desired number $Q$ of electrons. This removes the necessity of repeatedly solving the PTF equations for the self-consistent field, while studying the decay process.

\section{Quasibound states of the dot}

We construct the electron wave functions inside the dot in the envelope function approximation, using our parametrized potential, $U_{s}(x, y)$. The single electron energies are

$$
E_{n_{x}, n_{y}}=E_{n_{x}}+E_{n_{y}}
$$

and the electron wave functions factorize as

$$
\Psi_{n_{x}, n_{y}}(x, y)=\phi_{n_{x}}(x) \psi_{n_{y}}(y) .
$$

The factors satisfy 1D Schrödinger equations:

$$
\begin{aligned}
& -\frac{\hbar^{2}}{2 m^{*}} \phi_{n_{x}}^{\prime \prime}(x)+U(x) \phi_{n_{x}}(x)=E_{n_{x}} \phi_{n_{x}}(x), \\
& -\frac{\hbar^{2}}{2 m^{*}} \psi_{n_{y}}^{\prime \prime}(y)+W(y) \psi_{n_{y}}(y)=E_{n_{y}} \psi_{n_{y}}(y) .
\end{aligned}
$$

The second equation is for a confined wave function, easily solved by standard numerical methods. We label the solutions by the number of loops, $n_{y}$, of the eigenfunction. For example, taking $W(y)$ to have hard walls, the energy is

$$
E_{n_{y}, s w}=\frac{\hbar^{2}}{2 m^{*}}\left(\frac{n_{y} \pi}{w_{y}}\right)^{2} .
$$

For the truncated harmonic oscillator shape there is no similar analytic expression, but the dependence on $n_{y}$ is similar. 


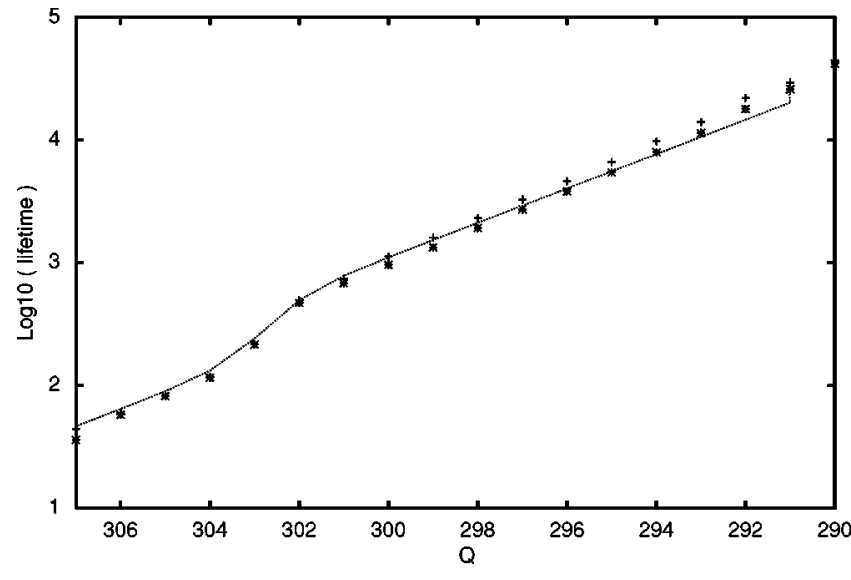

FIG. 6. Calculated lifetimes (in seconds) when $W(y)$ is either the truncated harmonic oscillator, stars; or a square well with $w_{y}$ $=380 \mathrm{~nm},+$ signs. The dotted line is the prediction of the two level model, Eq. (22).

The $x$-dependent equation describes 1D electrons confined in the dot by the "leaky barriers." Weakly quasibound state solutions were computed using methods described in Ref. 14 However, for levels corresponding to the long tunneling lifetimes observed in the experiment, the energies and eigenfunctions can be computed well enough by the simpler prescription of setting the electron wave function to zero at the points $\pm x_{b}$ inside the barriers. Furthermore, if only the eigenvalues and lifetimes are needed, we have checked that the WKB quantization condition is adequate:

$$
\int_{x_{l}}^{x_{r}} \sqrt{\frac{2 m^{*}}{\hbar^{2}}\left[E\left(n_{x}\right)-U(x)\right]} d x=\left(n_{x}-\frac{1}{2}\right) \pi .
$$

In the Appendixes we describe the determination of the lifetimes $\tau_{n_{x}}$. From here on the energies presented are obtained in the WKB approximation. The differences from the more accurate predictions using the true quasibound state energies can scarcely be seen on the scale of the graphs. For barrier penetrability we use Eq. (12).

We "construct' the desired dot configuration with excess electrons by generating a $U_{s}(x, y)$ for the chosen value of $Q$, and filling the levels as follows: (a) First we list the $\left(E_{n_{x}}, \tau_{n_{x}}\right)$, in order of increasing $n_{x}$ (and therefore of increasing energy and decreasing lifetime.) This list is truncated at an $n_{x}=n_{x, \max }$ whose lifetime is less than $0.01 \mathrm{sec}$. (b) Next we form a list of 2D levels $\left(n_{x}, n_{y}\right)$ by choosing those for which

$$
E_{n_{x}}+E_{n_{y}} \leqslant E_{n_{x, \max }}+E_{n_{y}=1} .
$$

The levels in this list are occupied in order of increasing energy and according to Fermi statistics; see Eqs. (A5) and (A6). We choose the dot Fermi level so that the number of electrons is the desired $Q$. It is supposed that, for the long lifetimes observed in the experiment, the electrons have time to lose energy by phonon collisions and occupy the quasibound states of lowest energy. Then, as described in Appendix A, we determine the lifetime for one electron to escape from the dot. This involves a weighted average of the level lifetimes, according to the occupancy of each level at the experimental temperature $T^{\prime}=100 \mathrm{mK}$.

To produce a sequence of decays for comparison to experiment we proceed as follows: (i) we start with a dot containing a number of electrons, $Q_{0}$, chosen large enough so that the lifetime for one electron to escape is smaller than those observed in experiment. (ii) We redetermine the barrier and dot configuration for $Q=Q_{0}-1$ electrons, as described in the above paragraph and determine again the corresponding lifetime for escape of one electron. This process is repeated to generate a sequence of decays that covers and extends beyond the range of lifetimes measured in experiment. From that list we choose as the first observed electron decay that corresponding to the $Q$ whose lifetime is the first to be larger than $t_{0}=25 \mathrm{sec}$.

\section{RESULTS AND DISCUSSION}

In Fig. 6 we show results from our model, using parameters chosen as described above, for a range of lifetimes extending over three orders of magnitude. The stars correspond to the truncated harmonic oscillator choice for $W(y)$, whereas the +'s are for the square well choice (with a value $w_{y}=380 \mathrm{~nm}$ chosen to optimize the agreement with the other prescription in the range of experimental lifetimes, from 10 to $1000 \mathrm{sec}$ ). One sees that the trends are very similar. For $Q$ in the neighborhood of 304, the predicted decay lifetimes fall in the experimental range.

As already mentioned in Sec. II, our PTF simulations predict $Q=286$ for the dot in equilibrium with the surrounding electron gas. This is also what we find with this separable model, as the curve of lifetimes shown in Fig. 6 extrapolates smoothly up to $Q=287$, for which we predict a lifetime of $\log _{10} \tau=5.2$, or $44 \mathrm{~h}$. After that, the Fermi level of the electrons inside the dot falls below that of the surrounding $2 \mathrm{DEG}$ and further decays are blocked. It should take almost two days for the dot to reach equilibrium with its surroundings.

Before attempting a more detailed comparison with the experimental data it is useful to examine the main features in our predicted sequences. First we focus on the linear behavior for values $Q<300$. (We have found similar behavior in other ranges of $Q$ when we use slightly different sets of parameters.) Such a linear dependence occurs when our model produces a sequence of decays dominated by those from a single 1D electron level, i.e., corresponding to a fixed value of $n_{x}$. To understand why, suppose that at zero temperature and for $Q$ electrons, the occupied level with shortest lifetime is $\left(n_{x, s}, n_{y}\right)$, and that $\left\{n_{x}^{\prime}, n_{y}^{\prime}\right\}$ are occupied levels with higher energy and longer lifetime (this requires that at least $n_{x}^{\prime}<n_{x, s}$ for longer lifetime and $n_{y}^{\prime}>n_{y}$ for higher total energy). When one forms the $Q-1$ electron configuration according to the rules explained above, one of the $\left\{n_{x}^{\prime}, n_{y}^{\prime}\right\}$ levels will be empty, whereas the level $\left(n_{x, s}, n_{y}\right)$ will again be filled. In more physical terms, all the electrons with energy above that of the level with shortest lifetime will lose energy by phonon collisions and fall into the leaky level, from which they finally escape. Since the lifetime does not depend on $n_{y}$, all the electrons with energy above that of the 


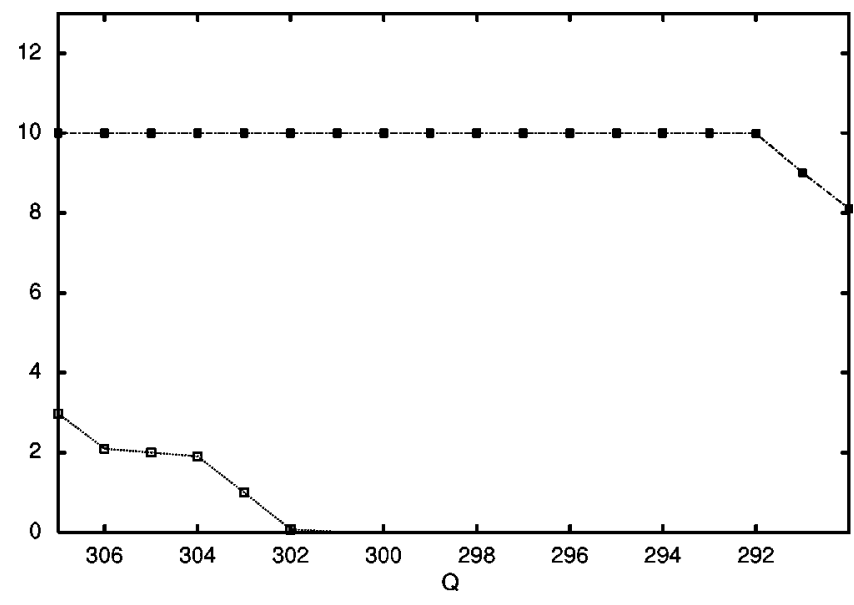

FIG. 7. Total occupation of levels with $n_{x}=13$ (black squares) and $n_{x}=14$ (open squares). The lines are drawn to guide the eye. The truncated harmonic oscillator model was used for $W(y)$.

state $\left(n_{x, s}, n_{y}=1\right)$ will escape through the same leaky 1D level, $n_{x, s}$, which remains the favored decay channel as long as it is occupied. Therefore the total probability for one electron to escape from the occupied states with quantum number $n_{x, s}$ is the probability for a single 1D electron with energy $E_{n_{x, s}}$, multiplied by the number $q_{n_{x, s}}$ of electrons in occupied states with the same quantum number $n_{x, s}$ :

$$
\tau(Q)=\frac{\tau_{n_{x, s}}(Q)}{q_{n_{x, s}}(Q)},
$$

and when the occupation $q_{n_{x, s}}$ of the leaky level is constant, the linear variation of $\log _{10}(\tau)$ reflects that of the lifetime of the leaky level. This is where the $2 \mathrm{D}$ nature of the quantum dot asserts its presence, even though the decay appears to proceed only in one dimension.

In Fig. 7 we show the occupations of the two levels with the shortest lifetimes. One sees that when $Q<300$ the occupation of the $n_{x}=13$ level stays practically constant and $n_{x}$ $=14$ level remains empty. For higher values of $Q$ both levels contribute significantly to the escape lifetime. In this situation,

$$
\tau(Q)=\frac{1}{q_{a}(Q) / \tau_{a}(Q)+q_{b}(Q) / \tau_{b}(Q)} .
$$

This is shown as the dotted curve in Fig. 6, and it accounts very well for the trend of the lifetimes predicted by the separable model.

Our separable model favors the appearance of the linear decay sequences because of the degeneracy in lifetime of states with the same $n_{x, s}$. A nonseparable model would lift that degeneracy and then the lifetime sequences should show a behavior intermediate between the two situations discussed above. In particular, the sudden change of slope at $Q=302$ in Fig. 6 would presumably spread over a wider range of values of $Q$. Not surprisingly, the predicted lifetimes for the observed decays depend sensitively on details of the barrier shape. Those shown in Fig. 8 correspond to the square well

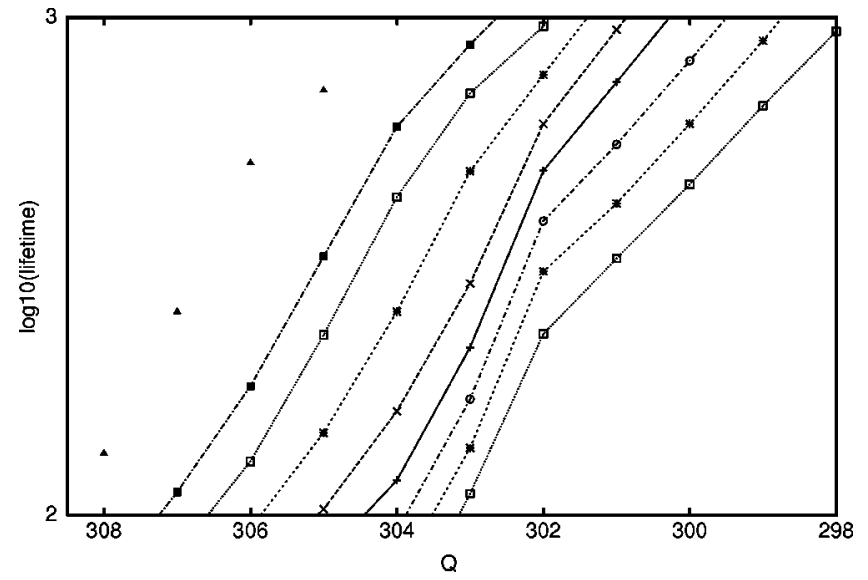

FIG. 8. Slow decays: Calculated lifetime sequences corresponding to variations of the standard barrier width from $-3 \%$ (right) to $+4 \%$ (left) in steps of $1 \%$. The + signs joined by a continuous line (to guide the eye) correspond to the prediction for the standard set of parameters. Experimental points (left triangles) taken from Fig. 1 with the origin of $Q$ shifted arbitrarily.

choice for $W(y)$ and our standard set of parameters. In addition we show how the lifetimes vary when the barrier width is changed by amounts ranging from $+4 \%$ to $-3 \%$ (from left to right). As can be seen, the exact value of each decay lifetime depends quite strongly on the barrier width, as expected for a tunneling process. But the number of slow decays is much more stable: four or five in most of the cases shown, and in several cases their lifetimes, are quite compatible with the experimental points. In particular it is remarkable that a $2 \%$ increase in the standard barrier width produces a sequence (third line from left) in excellent agreement with experiment (disconnected points shifted to extreme left).

There is a clear distinction between the lifetime trends of the thicker and thinner barrier widths. In the latter one sees very clearly the transition between escape from the $n_{x}=13$ and the $n_{x}=14$ levels at $Q=302$. For the thicker barrier widths, escape is dominated by the $n_{x}=14$ levels that become progressively more occupied above $Q=302$.

We have explored the dependence of the model predictions on changes by similar percentages of the barrier heights, potential floor $U_{0}$, and the width $w_{y}$ of $W(y)$. The results are qualitatively similar to those shown above for the changes in the barrier width, with a number of slow decays ranging from 4 to 6 , and in some cases they are very similar to the data in Fig. 1. We therefore conclude that our model predictions are quite consistent with the experimental trends, although a quantitative comparison with the measured lifetimes is hampered by the strong sensitivity of tunneling to any small change in the barrier shape.

Finally we show in Fig. 9 predictions for the fast decays: their number and location in a graph such as that of Fig. 1 depends very sensitively on the time $\left(t_{0}\right)$ beyond which the experiment measures lifetimes. That is, as the dot is isolated there must be a burst of very short-lived escapes, but after some seconds one reaches the stage where separate events can be recorded and the lifetimes deduced. The two dashed 


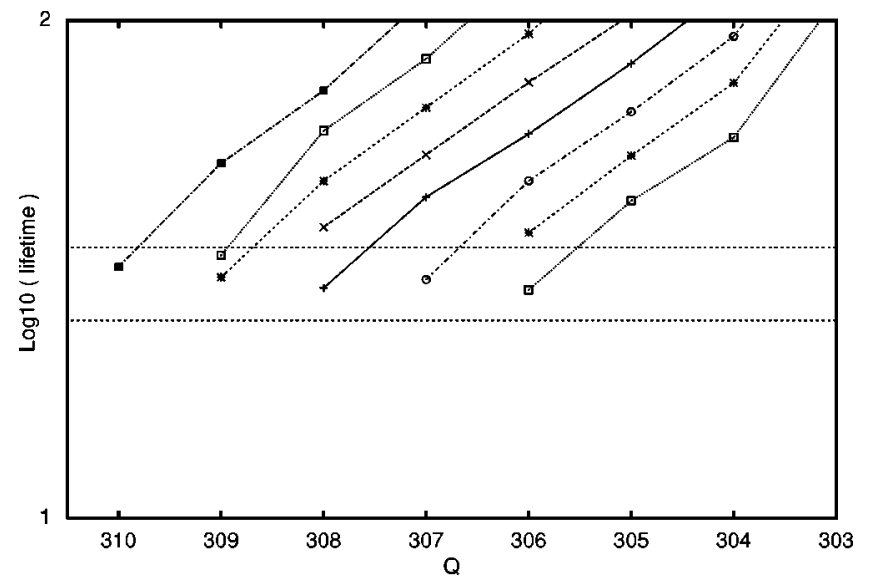

FIG. 9. Same as Fig. 8, but for the fast decays.

horizontal lines in Fig. 9 correspond to values of $\log _{10}(25 \mathrm{~s})$ and $\log _{10}(35 \mathrm{~s})$. As can be seen, when we explore the same range of barrier widths as in Fig. 8, the number of fast decays above that $t_{0}$ varies from 3 to 4 . The overall trend seems to be consistent with experiment, in particular if the value of $t_{0}$ is increased towards the more pessimistic estimate of $35 \mathrm{~s}$.

\section{SUMMARY AND CONCLUSIONS}

Electron escape from a strongly isolated dot with excess electrons has been studied in the framework of the selfconsistent Poisson-Schrödinger and Poisson-Thomas-Fermi approximations. Based on these calculations a rectangular separable potential model has been devised that incorporates the main features of the self-consistent field. Rearrangement effects are taken into account by recalculating the confining potential $U_{s}(x, y)$ after each electron escape.

The use of a separable potential introduces certain correlations in the energy spectrum of the single-electron orbitals. A more realistic confining potential would have a more rounded shape, which would remove the separability and modify those correlations. In the same vein, the tunneling in our simplified model is $1 \mathrm{D}$, whereas the actual process is $2 \mathrm{D}$.

We find it quite remarkable that despite all these simplifications the predictions turn out to be so satisfactory. The model therefore may be reliable for extrapolating to longer times. For instance, we find that the isolated dot would hold one excess electron for as long as $44 \mathrm{~h}$. On such a time scale, one could use well isolated dots containing a few long-lived electrons to study their entangled states. This would open an interesting new approach to the implementation of quantum computation in semiconductor devices.

\section{ACKNOWLEDGMENTS}

We are grateful to DGES-Spain for continued support through Grants No. PB97-0915 and No. UE97-0014 (J.M.), to EPSRC-UK (C.S.), and to NSERC-Canada for Research Grant No. SAPIN-3198 (D.W.L.S.). This work was carried out as part of QUADRANT, Esprit Project No. EP-23362 funded by the E.U.

\section{APPENDIX A: LIFETIMES}

We summarize here the expressions relating the lifetimes to the probability of transmission across the barrier. We follow the standard treatment and definitions for $\alpha$-particle decay in nuclear physics, as can be found, for example, in Ref. 4.

Our potential $U_{s}(x, y)$ is separable, and the electron can escape only across the barriers in the $x$ direction. Therefore, we have adapted the expressions derived in Ref. 4 to the 1D situation.

The lifetime $\tau=1 / \lambda$ is the inverse of the "decay constant," defined as the number of "decays" per second per parent "dot." For one dot the electron wave function is normalized to unity over the volume inside the barriers, and $\lambda$ for a given level is just the outgoing flux at large distance.

When the decay probability is small, one can treat the electron as confined in the dot. Classically, its trajectory will oscillate between the right, $x_{r}$, and left, $x_{l}$, turning points, with a period

$$
P=2 \int_{x_{r}}^{x_{l}} \frac{d x}{v(x)},
$$

where $v(x)$ is the classical electron velocity at energy $E_{x}$ :

$$
v(x)=\sqrt{\frac{2}{m^{*}}\left[E_{x}-U(x)\right] .}
$$

The flux $\lambda$ is then given by the frequency of hits against the barriers, $2 / P$, times the transmission probability $T$ across a barrier, and therefore

$$
\tau=\frac{1}{\lambda}=\frac{1}{T} \int_{x_{l}}^{x_{r}} \frac{d x}{v} .
$$

This expression is very convenient because the transmission coefficient Eq. (12) for our parametrized potential, $U(x)$, is known analytically. ${ }^{13}$ For more general barrier profiles and the long lifetimes of interest, one can use the WKB approach and its corresponding connection formulas across the barrier (see, e.g., Appendix D of Ref. 4):

$$
\begin{gathered}
T_{W K B} \approx e^{2 \omega} \\
\omega=\int_{x_{r}}^{x_{t}} \kappa d x=\int_{x_{r}}^{x_{t}} \sqrt{\frac{2 m}{\hbar^{2}}\left[U(x)-E_{x}\right]} d x .
\end{gathered}
$$

If the WKB wave function is used inside the well to determine the period $P$, the same decay half-life is obtained as in Eq. (A3) above.

Since the dot is located inside a crystal at temperature $T^{\prime}$, via phonon coupling the electrons in the dot should also be at the same temperature. The level occupations $f(E)$ are determined by Fermi statistics:

$$
f(E)=\left[1+e^{\left(E-E_{F}\right) / k_{B} T^{\prime}}\right]^{-1},
$$

where these are now 2D energies. The Fermi level is obtained from 


$$
Q=\sum_{i=\left(n_{x}, n_{y}\right)} 2 f\left(E_{i}\right)
$$

where the factor of 2 accounts for spin degeneracy. For the ensemble of electrons in the dot, the flux $\lambda$ will now be the sum of fluxes for each occupied single-particle level, weighted by the level occupancy:

$$
\lambda=\sum_{i=\left(n_{x}, n_{y}\right)} 2 f\left(E_{i}\right) \lambda_{i},
$$

and the corresponding half-life is still $\tau=1 / \lambda$. In particular this argument applies in the $T^{\prime}=0$ limit, as we implicitly assumed in Sec II to explain the sequence of lifetimes.

\section{APPENDIX B: LIFETIME DEPENDENCE ON $Q$}

For a level of given $n_{x}$, the lifetime depends on $Q$ because the barrier characteristics change as does the level energy $E_{n_{x}}$. The latter varies mainly because $U_{0}$ depends on $Q$, and this affects the transmission probability $T$. To good approximation

$$
\frac{d E_{n_{x}}}{d Q} \simeq \frac{d U_{0}}{d Q} .
$$

Neglecting the dependence of the level lifetime on the period $P$, we can write

$$
\frac{d \ln \tau}{d Q} \simeq-\frac{d \ln T}{d E} \frac{d E}{d Q} .
$$

Taking the transmission probability $T$ from the WKB expression leads to

$$
\begin{aligned}
\frac{d \ln \tau}{d Q} & =2 \frac{d}{d Q} \int_{\text {barrier }} \sqrt{\frac{2 m^{*}}{\hbar^{2}}[U(x)-E]} d x \\
& =\int_{\text {barrier }} \sqrt{\frac{2 m^{*}}{\hbar^{2}} \frac{1}{U(x)-E}\left(\frac{d U(x)}{d Q}-\frac{d E}{d Q}\right) d x .}
\end{aligned}
$$

Noting Eq. (B1), the second contribution to the integral depends linearly on the placement of the potential floor.

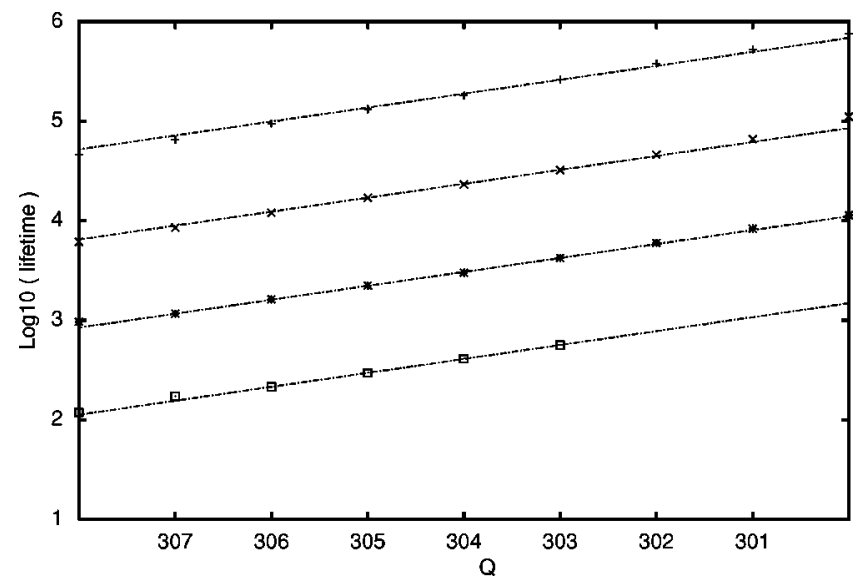

FIG. 10. Dependence of lifetime on $Q$ for the occupied levels with $n_{x}=11,+$ signs; $n_{x}=12, \times$ signs; $n_{x}=13$, stars; and $n_{x}$ $=14$, open squares. The dash-dotted lines correspond to Eq. (B5).

However, the variation of the barrier shape $[d U(x) / d Q]$ cannot be neglected. Indeed, Eq. (8) gives approximately

$\frac{d U(x)}{d Q}=\frac{d U_{0}}{d Q}\left(\frac{1}{3}+\frac{2}{3} \frac{\sinh ^{2}\left(\frac{x-x_{b}}{w_{b}}\right)}{e^{-2 \mu} \cosh ^{2}\left(\frac{x-x_{b}}{w_{b}}-\mu\right)}\right), \quad x<x_{b}$

$\frac{d U(x)}{d Q}=\frac{d U_{0}}{d Q}\left(\frac{1}{3}-\frac{1}{3} \frac{\sinh ^{2}\left(\frac{x-x_{b}}{w_{b}}\right)}{e^{2 \mu} \cosh ^{2}\left(\frac{x-x_{b}}{w_{b}}-\mu\right)}\right), \quad x>x_{b}$

Using Eq. (B4), the contribution from $d U / d Q$ to the integral of Eq. (B3) is obtained with an accuracy better than $2 \%$.

For the standard choice of parameters, and $Q$ in the range 300 to 310 the computed values of $d \log _{10} \tau / d Q$ turn out to be $\simeq-0.14$ for the levels of interest. In Fig. 10 we plot the evolution of the level lifetimes with $Q$, compared to the expression $\left(Q_{0}=303\right)$

$$
\log _{10} \tau_{n_{x}}(Q)=\log _{10} \tau_{n_{x}}\left(Q_{0}\right)-0.14\left(Q-Q_{0}\right)
$$

${ }^{1}$ J. Cooper, C.G. Smith, D.A. Ritchie, E.H. Linfield, Y. Jin, and H. Launois, Physica E (Amsterdam) 6, 457 (2000).

${ }^{2}$ G. Gamow, Z. Phys. 51, 204 (1928).

${ }^{3}$ E.U. Condon and R.W. Gurney, Nature (London) 122, 439 (1928).

${ }^{4}$ M.A. Preston and R.K. Bhaduri, Structure of the Nucleus (Addison-Wesley, Reading, MA, 1975), Part III and Appendix D.

${ }^{5}$ W. van Dijk and Y. Nogami, Phys. Rev. Lett. 83, 2867 (1999).

${ }^{6}$ J. Martorell and D.W.L. Sprung (unpublished QUADRANT progress reports).

${ }^{7}$ J. Martorell and D.W.L. Sprung, Phys. Rev. B 49, 13750 (1994).
${ }^{8}$ J. Martorell, Hua Wu, and D.W.L. Sprung, Phys. Rev. B 50, 17298 (1994).

${ }^{9}$ W. Mönch, Semiconductor Surfaces and Interfaces (Springer, Berlin, 1993).

${ }^{10}$ J.H. Davies, Semicond. Sci. Technol. 3, 995 (1988).

${ }^{11}$ J.H. Davies, A. Larkin, and E.V. Sukhorukov, J. Appl. Phys. 77, 4504 (1995).

${ }^{12}$ J. Martorell and D.W.L. Sprung, Phys. Rev. B 54, 11386 (1996).

${ }^{13}$ P.M. Morse and H. Feshbach, Methods of Theoretical Physics (McGraw Hill, New York, 1953), pp. 1651-1660.

${ }^{14}$ J. Killingbeck, J. Phys. A 10, L99 (1977); Phys. Lett. 78A, 235 (1980). 